\title{
Late Onset Recurrent Syncope after Permanent Pacemaker Implantation
}

\author{
Steven Mazzone ${ }^{1}$, Utkarsh Kohli ${ }^{1}$, Zaid Aziz ${ }^{1}$, Andrew Beaser ${ }^{1}$, Gaurav Upadhyay ${ }^{1}$, \\ Roderick Tung ${ }^{1}$, and Hemal Nayak ${ }^{1}$ \\ ${ }^{1}$ University of Chicago
}

July 9, 2020

\section{Case Presentation}

A 62 year old man with a past medical history of hypertension and mild coronary artery disease underwent dual chamber permanent pacemaker (PPM) implantation in April of 2015 for complete heart block. A Boston Scientific Ingenio K173 (Boston Scientific, St. Paul, MN) generator was inserted and connected to 2 Medtronic 5076 pacing leads (Medtronic, Minneapolis, MN) which were positioned in the right atrium (RA) and right ventricle (RV). The operative report noted "normal testing results" with an acute RV lead bipolar pacing threshold of $0.6 \mathrm{~V}$ at $0.4 \mathrm{msec}$ and pacing impedance of $550 \Omega$. The PPM was programmed DDDR 60-130 bpm with RA and RV lead outputs programmed 3 times threshold.

He had no further syncope after implant but continued to complain of intermittent lightheadedness as soon as a week out from the implant. Multiple pacemaker interrogations revealed stable atrial and ventricular lead function and he was told that his symptoms were likely due to low blood pressure which resulted in his blood pressure medicines being adjusted. The patient presented to his local hospital with recurrent syncope without warning 14 months after the initial implant. PPM interrogation recorded noise resulting in RV pacing inhibition (Figure 1). RV bipolar lead pacing threshold was $0.6 \mathrm{~V}$ at $0.4 \mathrm{msec}$ and the pacing impedance measured $660 \Omega$. Historical RV lead pacing threshold and impedance trends were stable. RV lead oversensing was suspected and pocket manipulation, deep inspiration, Valsalva maneuver, arm isometrics, left arm extension, abduction and adduction did not duplicate the noise. The device was reprogrammed to asynchronous mode (DOO at $80 \mathrm{bpm}$ ) and the patient was transferred to our hospital for lead extraction.

After confirming the interrogation findings, a chest radiograph was performed which showed no obvious fracture, gross dislodgement or lead discontinuity. What is the likely cause of the noise on the RV lead and this patient's recurrent syncopal episodes?

\section{Discussion}

PPM malfunction is an infrequent cause for recurrent syncope. ${ }^{1} \mathrm{PPM}$ malfunction due to electrical connection problems such as a loose set screw or lead-header malapposition, is distinctly uncommon. ${ }^{2,3,4,5}$

The chest radiograph revealed that the RV lead terminal pin was not fully inserted into or engaged in the pacemaker header (Figure 2). This was suspected as the potential cause for the intermittent noise. The patient was brought to the electrophysiology laboratory for lead revision. Failure of complete lead terminal pin insertion was confirmed intra-op (Figure 3). Both set screws were set but the distal set screw was not fully contacting the terminal pin. The RV lead was extensively tested using the pacing system analyzer revealing a bipolar threshold of $0.6 \mathrm{~V}$ at $0.4 \mathrm{msec}$ and a pacing impedance of 630-660 $\Omega$. New lead insertion or lead extraction was not performed and the RV lead was fully reinserted into the header and both set screws were tightened. At 22 months follow-up, the patient is asymptomatic without syncope or lightheadedness 
and device function is normal.

Oversensing in cardiovascular implantable electronic devices (CIEDs) can be defined as sensing of signals that are not caused by local depolarizations and is often due to physiologic or non-physiologic causes ${ }^{2}$. Nonphysiologic causes of oversensing, often referred to as noise, include electromagnetic interference (EMI), lead failure, lead-header connection problems, myopotentials, and sensing of extraneous pacemaker signals, such as those emitted by the minute ventilation sensor. ${ }^{6,7}$

Determining the cause of noise requires a step-by-step approach and often involves a process of elimination. For example, if noise is due to EMI (external source), it should be recorded on all channels which was not the case in our patient. Lead component failure (fracture or insulation breach) is the most common cause of non-physiologic oversensing and is often associated with changes in impedance, sensing or threshold. ${ }^{2}$ Lead parameters were stable in our patient. Oversensing of myopotentials (either due to diaphragm or pectoral muscle) is often provocable with maneuvers such as deep inspiration, isometrics, or straining. ${ }^{3}$ None of these maneuvers elicited noise in our patient. Noise due to oversensing of minute ventilation signals are also high frequency, intermittent and may resemble those seen in our patient, but the Ingenio pacemaker has not been involved in the recent Boston Scientific safety advisory. ${ }^{8}$

Lead-header connection problems such as loose set screw or terminal pin-header malapposition most often present soon after implantation. Our case is unusual in its late presentation resulting in recurrent syncope 14 months after the initial implant. Because noise due to lead-header connection problems is rare, it is often overlooked, but can be recognized by its characteristic appearance - typically high frequency, intermittent, with saturation of the amplifier. ${ }^{9,}{ }^{10}$ Because of the intermittent make-or-break behavior of the connection problem, changes in impedance, sensing and threshold may not always be present.

Recurrent syncope after PPM implantation is a common problem, often leading to emergency room visits, but is rarely due to PPM malfunction. In a study evaluating 162 patients presenting with syncope after PPM implantation, device malfunction was responsible for less than $5 \%(8 / 162)$ of the episodes. ${ }^{1}$ Premature battery or primary lead failure were responsible for all 8 cases and required either generator change or new lead insertion. No lead-header connection problems were noted. The etiology of the syncope was not found in $>50 \%$ of patients in this cohort but hemodynamic causes such as orthostasis were suspected.

The evaluation of a patient who presents with recurrent syncope after CIED implantation includes device interrogation in addition to orthostatic vital signs, electrocardiogram and routine laboratory data. ${ }^{1}$ Chest radiography (CXR) is often performed in the evaluation of PPM malfunction. While not systematically studied, the reported yield of routine CXR is low. ${ }^{1,11}$ This is likely due to the fact that a lead insulation breach, micro-fracture or micro-dislodgement are usually not visible on CXR. However, in our patient, the CXR was valuable in helping find the cause for the noise. Since CXR is a modality that is readily available, non-invasive and relatively inexpensive, it has value and should be performed in the evaluation of PPM malfunction.

\section{References}

1. Ofman P, Rahilly-Tierney C, Djousse L, Peralta A, Hoffmeister P, Gaziano JM, Weiss A, Lotan C, Rosenheck S. Pacing system malfunction is a rare cause of hospital admission for syncope in patients with a permanent pacemaker. Pacing Clin Electrophysiol. 2013;36:109-112.

2. Gunderson BD, Swerdlow CD, Wilcox JM, Hayman JE, Ousdigian KT, Ellenbogen KA. Causes of ventricular oversensing in implantable cardioverter-defibrillators: implications for diagnosis of lead fracture. Heart Rhythm. 2010;7:626-633.

3. Sondhi S, Bhardwaj R, Kandoria A, Ganju N and Sharma R. Pacemaker malfunction - Disengagement of lead PIN from connectorhead - Rare cause for loss of capture. IHJ Cardiovascular Case Reports (CVCR). 2018; 2:196-200.

4. Coleman AE, Defrancesco TC, Chanoit G. Pacemaker malfunction due to mechanical failure of the lead-header interface. $J$ Vet Cardiol. 2012;14:519-523. doi:10.1016/j.jvc.2012.07.003 
5. Kuruvilla C, Voigt L, Kachmar K, Reddy CV, Kassotis J. Inappropriate mode switching in a dual chamber pacemaker due to oversensing of a high frequency signal from a conductor/ring discontinuity (loose set screw). Pacing Clin Electrophysiol. 2002;25:115-117. doi:10.1046/j.1460-9592.2002.00115.x

6. Kowalski M, Ellenbogen KA, Wood MA, Friedman PL. Implantable cardiac defibrillator lead failure or myopotential oversensing? An approach to the diagnosis of noise on lead electrograms. Europace 2008; 10:914-917

7. McClelland I, Nayak HM, Tung R, Upadhyay GA. Respiratory rate trending as a cause for atrial lead noise: a first report in an implantable defibrillator patient. Heart Rhythm Case Reports 2018;4:454-457

8. Minute Ventilation Signal Oversensing Physician Letter. December 2017, 2018. http://www.bostonscientific.com/en-US/pprc/product-advisories.html

9. Swerdlow CD, Friedman PA. Advanced ICD Troubleshooting: Part I. Pacing Clin Electrophysiol. 2005;28:1322-1346

10. Mulpuru SK, Madhavan M, McCleod CJ, Cha Y and Friedman PA. Cardiac pacemakers: function, troubleshooting and management. J Am Coll Cardiol. 2017;69:189-210

11. Pavlovic SU, Kocovic D, Djordjevic M, Belkic K, Kostic D, Velimirovic D. The etiology of syncope in pacemaker patients. Pacing Clin Electrophysiol. 1991;14:2086-2081.

\section{Figure Legend}

\section{Figure 1: Stored electrograms at the time of the syncopal episode}

Stored electrograms corresponding to the time of the patient's syncopal episode showing high frequency, nonphysiologic (cycle length $=150 \mathrm{msec}$ ) noise on the ventricular $(\mathrm{V})$ channel which saturates the amplifier. The pacemaker interprets the noise as ventricular tachycardia (VT) on the marker channel (M). There is pacing inhibition (no ventricular pace $[\mathrm{VP}]$ ) is noted). $\mathrm{A}=$ atrial channel, $\mathrm{V}=$ ventricular channel, $\mathrm{M}=$ marker channel, $\mathrm{VT}=$ ventricular tachycardia, $\mathrm{AS}=$ atrial sense, $\mathrm{PVP}=$ Post ventricular atrial refractory period extension

\section{Figure 2: Magnified view of the pacemaker generator on chest radiography}

Antero-posterior chest radiograph with a magnified view of the pacemaker generator showing that the ventricular lead is not fully inserted into the header and the terminal pin is not seen past the distal set screw (black arrow). In contrast, the atrial lead is fully inserted.

\section{Figure 3: Intra-operative photo confirming lead-header malapposition}

Photograph of the generator taken at the time of the lead revision procedure confirming that the ventricular lead terminal pin was not completely or fully inserted into the header (white arrow). A = atrial lead port, $\mathrm{V}=$ ventricular lead port

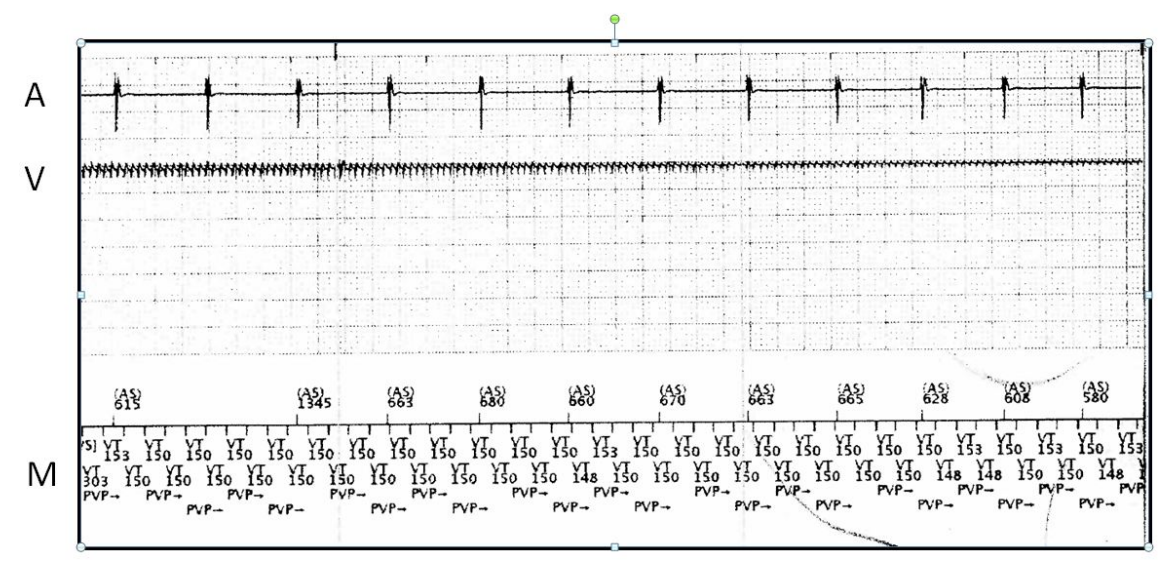



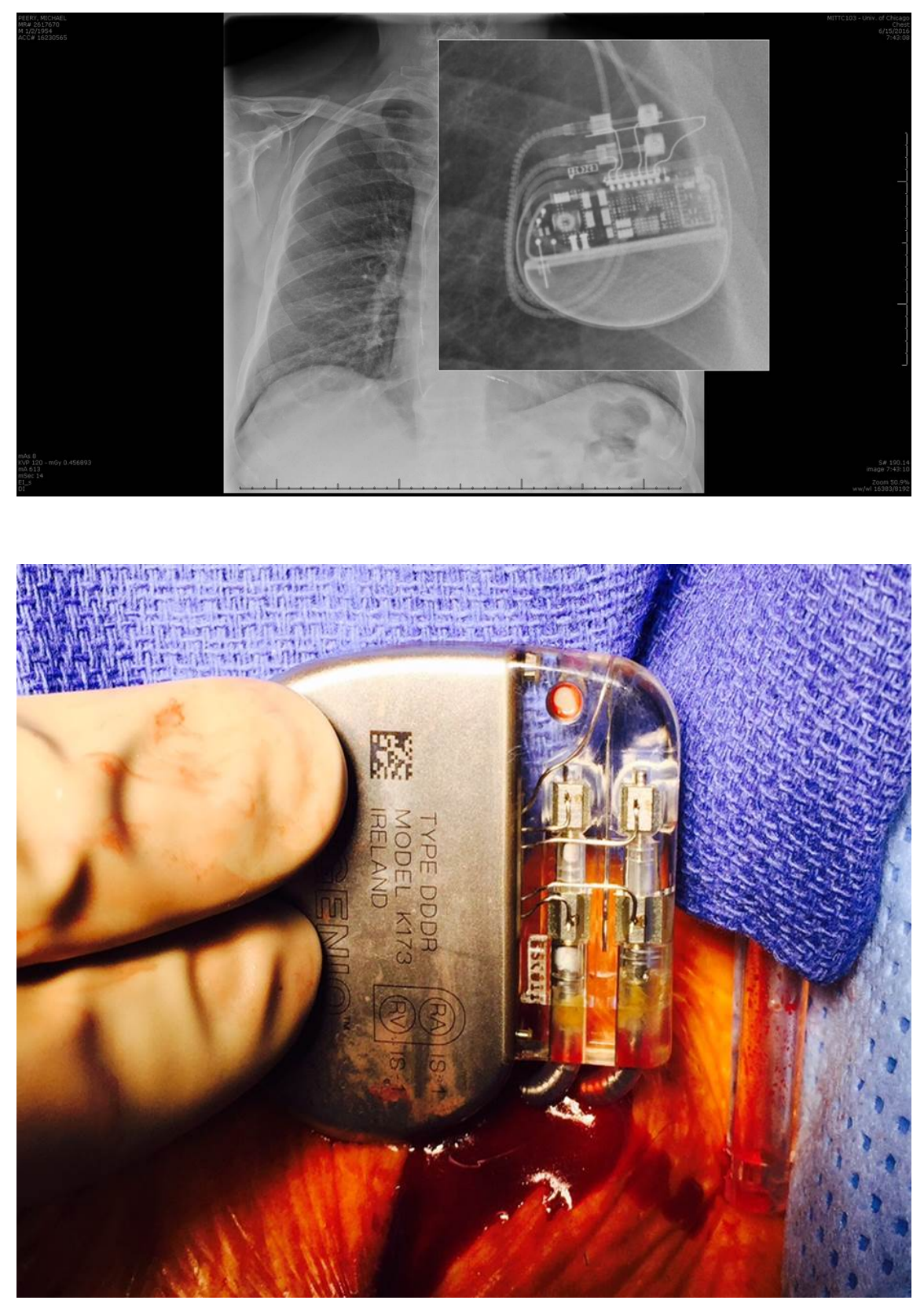